\title{
Overwhelming Flow of Water using Machine Learning Techniques
}

\author{
Savithri, Suganya.D
}

\begin{abstract}
Floods are rare and dangerous disaster in minimum duration, which have the most destructive impact within urban and rural areas. This research in flood prediction models contributed to risk reduction, to prevent the loss of human life, and reduce the property of damage in floods. This study implements the automated machine learning models, using the Support Vector Machine (SVM) and Artificial Neural Network $(A N N)$. The rainfall data and various meteorological parameter which include temperature data are used in this study. Concurrent daily records of inflow and discharge are taken into consideration to calculate the water level to quantify the importance of the lake flow. It aims to discovering accurate and efficient for the flood forecasting model. This paper attempts to forecast flood by modelling water level, temperature and rainfall data in the region of Korattur lake, Chennai, India. In this study, ultrasonic sensor used to capture the measurement of water level to predict from ultrasonic waves and input of same implemented in BPNN and Support Vector Machine (SVM) were used for flood forecasting. The water level flow is deducted in this research using ultrasonic sensor, proves the best efficient models applied for flood forecasting. This study can be used as a predicting the flood by choosing the proper Machine Learning (ML) algorithm such as Support Vector Machine (SVM) and Artificial Neural Network (ANN) algorithm for showing higher accuracy. To get more accurate result of the models, three standard statistical performance evaluation parameters, Root Mean Square Error (RMSE), Mean Absolute Error (MAE) and coefficient of determination $\left(R^{2}\right)$ were used to analyse the performance of the model developed. As a result, the proposed model proves the most efficiency and accuracy for predicting the flood forecasting.
\end{abstract}

Keywords: ultrasonic waves, metrological parameter, ultrasonic sensor

\section{INTRODUCTION}

Flood are the most dangerous disaster to the people and nature. Floods occur every year in every part of India. In southern India, they have terrible flood by the rise of water in the rivers. Floods make many people lose their resources and destroy crop in the fields and cause famine. To prevent the flood, many methods are taken from the government. Some steps are derived from technical aspects. With the evolution of computer developing computation model for the purpose of flood forecasting become more accurate. In this paper, the study on Artificial Intelligence (AI) in Machine Learning (ML) helps to predict the flood forecasting. Machine Learning has the ability to adapt to new circumstance and to

Revised Manuscript Received on December 30, 2019.

Dr.Savithri, Professor, Department of Computer Science, Women's Christian College, Chennai, Tamilnadu, India Email: dr,savithri.v@gmail.com Women's Christian College, Chennai, Tamilnadu, India Email: savi3_8@yahoo.co.in
D.Suganya, Student, Department of Computer Science,

detect and recognize pattern. The scope of this research is applied on application of machine learning in artificial intelligence. Today's major flood prediction models are mainly data-specific and include various simplified assumptions. There are numerical weather prediction model have improved their performance, but they are not unable to provide accurate models for expected resolution. A comparison of Machine Learning (ML) techniques Artificial Neural Network (ANN) and Support Vector Machine (SVM) has been taken into consideration to predict the flood forecasting for higher accuracy. In recent years, Artificial Neural Network (ANN) is the main algorithm of Machine Learning (ML) has widely used in developing the predictive models. ANN is a subset of machine learning. ANNs are used in prediction, classification and clustering. Another main algorithm of machine learning is Support Vector Machine (SVM). This technique for classifying and regression and also employs a hyper plan to separate data points. Machine learning algorithms have important characteristics that need to be carefully taken into consideration. Finally, the effective algorithm to build the classification model and the most influential attributes for flood detection were defined.In this paper, weather related historical year wise data of Chennai district in South India are provided. To Predict the flood, water flow is an important aspects of water resources management. This water flow considering inflow and discharge of the river data are collected. The temperature and humidity data have chosen to determine the atmospheric stability.

The objectives are

- The main objectives of this research is to analyse the flood forecasting from the Red hills lake,Chennai. In details, Artificial Neural Network (ANN) and Support Vector Machine (SVM) model are being initiated.

- The proposed study is used to determine the water level using ultrasonic sensor. This technology helps to predict flood through ultrasonic waves. This system analysed to measure the performance evaluation for better accuracy in flood prediction.

- The systematic approach has developed to detect water level from ultrasonic sensor and the same compared and validated using ANN and SVM model.

\section{LITERATURE REVIEW}

A thorough study of several paper based on the Artificial Neural Network (ANN), Support Vector Machine (SVM) and Back Propagation (BP) algorithm to understand the 
prediction of flood in machine learning using artificial intelligence. In [1], Active Learning Method (ALM) is compared with Support Vector Machine (SVM) to predict long term simulation of daily stream flow in river. The daily discharge data were utilized for training and testing of the models. It results SVM is a well-known method for run-off simulation and its capabilities have been demonstrated. In [2], aimed to forecast the River Nile in Sudan using an Artificial Neural Network (ANN). This model validates the accuracy against the actual flow. This analysis indicates that ANN provide a reliable flood detecting in the River Nile. In [3], authors explore of machine learning methods for flood forecasting in river. This analysis based on several machine learning algorithm and set of upstream and downstream flood was tested. Its results Bayesian Linear model used for forecasting of extreme flood events. In [4], authors present the knowledge of current and emerging trends in Artificial Neural Network (ANN) application research. This paper proposed feed forward and feedback propagation ANN model for research based on data analysis like accuracy, performance, volume, convergence and processing speed. In [5], authors have proposed a two-level approach for clustering large data set for rainfall data prediction with Support Vector Machine (SVM). In this approach perform well direct clustering and to reduce the computation time.In [6], authors have proposed Artificial Neural Network (ANN) are component to improve rainfall forecast. This technique was used to provide more monthly rainfall forecast and it has an application where there is temperature and rainfall data.In [7], authors propose a deep learning approach by integrating Stacked Auto Encoders (SAE) and Back Propagation Neural Network (BPNN) for the prediction of stream flow. This experiment research result in SAE-BP integrated algorithm perform much better. In [8], Artificial Neural Network (ANN) model were applied in River to forecast the flood. The observation of data were river-stage and rainfall are validated since the river-stage observation has started. The input and output data of the neural network in river-stage predicted that it has very good
accuracy.In [9], authors describe Artificial Neural Network (ANN), feed-forward back propagation networks, radial basis network used for modelling the stream flow. This result ANN has the capacity to forecast the stream flow of the river and hydrological parameters are difficult to obtain.In [10], authors propose a flood forecasting model is present that exploits the real time information and to predict the water-level evolution. Accurate prediction is obtained using a two-year data set of input data. It results, as per the flow-rate increase in the data, the accuracy of prediction increases. In [11], Linear Least Square and Simplex optimization (LLSSIM) is used to examine the ability of a three-layer feed forward Artificial Neural Network (ANN) to apply in various climate regimes. In this ANN model capable of simulating well the daily high and low flows and validating pseudo-precipitation.In [12], here the Average Mean Square Error (AMSE) for each kernel is evaluated and the kernel having minimum Mean Square Error (MSE) is selected for rainfall prediction. It results in Support Vector Machine (SVM) model is polynomial kernel and has low MSE among alIn [13], authors propose Artificial Neural Network (ANN) model for the prediction of flooding in Nigeria using deep feed-forward neural network. The dataset was used to train the network using the back-propagation algorithm. This result in average accuracy of the model in flood prediction.In [14], Dynamic Regression Model which is statistically based model for historical flows and climate data of the basin to generate a forecast. Artificial Neural Network (ANN) model for river flow forecasting and also considered two ANN model which is General Regression Neural Network (GRNN) and Back Propagation Neural Network (BPNN).In [15], Feed Forward Neural Network (FFNN) model is used to prediction rainfall data. The input layer indicates the past values and the output layer indicates the current values. Back propagation algorithm is applied in training and validating process. The prediction result be evaluated in Least Mean Square Error (LMSE).

\section{III.DATA COLLECTION}

The table predicts the average rainfall in the years

\begin{tabular}{|l|l|l|l|l|l|l|l|l|l|l|}
\hline \multirow{2}{*}{ YR } & $\begin{array}{l}\text { A.R: } \\
\text { JUL }\end{array}$ & $\begin{array}{l}\text { A.R: } \\
\text { AUG }\end{array}$ & $\begin{array}{l}\text { A.R: } \\
\text { SEP }\end{array}$ & $\begin{array}{l}\text { A.R: } \\
\text { OCT }\end{array}$ & $\begin{array}{l}\text { A.R: } \\
\text { NOV }\end{array}$ & $\begin{array}{l}\text { E.R: } \\
\text { JUL }\end{array}$ & $\begin{array}{l}\text { E.R: } \\
\text { AUG }\end{array}$ & $\begin{array}{l}\text { E.R: } \\
\text { SEP }\end{array}$ & $\begin{array}{l}\text { E.R: } \\
\text { OCT }\end{array}$ & $\begin{array}{l}\text { E.R: } \\
\text { NOV }\end{array}$ \\
\hline 1901 & 155.8 & 191.5 & 138.4 & 137.1 & 622.9 & -1.7 & -10.1 & -18.5 & -15.1 & -11.4 \\
\hline 1902 & 118.9 & 214.4 & 159.7 & 193.7 & 686.7 & -25.3 & 0.2 & -6.2 & 19.9 & -2.6 \\
\hline 1903 & 141 & 300.2 & 206.2 & 212.5 & 859.9 & -12 & 39.6 & 20.7 & 31.3 & 21.5 \\
\hline 1904 & 191.9 & 191.4 & 89.5 & 130.6 & 603.5 & 19.4 & -11.1 & -47.6 & -19.3 & -14.9 \\
\hline 1905 & 153.5 & 120.6 & 187.6 & 99 & 560.6 & -4 & -43.1 & 10.4 & -38.6 & -20.3 \\
\hline 1906 & 174 & 219.9 & 221.8 & 110.8 & 726.5 & 8.6 & 3.1 & 30.7 & -31.3 & 3.1 \\
\hline 1907 & 184.8 & 206.3 & 216.3 & 129.3 & 736.8 & 14.7 & -4 & 26.5 & -20.1 & 3.9 \\
\hline 1908 & 125 & 240.1 & 155.1 & 247.6 & 767.7 & -22.7 & 11.6 & -9.1 & 53.3 & 8.3 \\
\hline 1909 & 157.3 & 227.5 & 196 & 148.2 & 729 & -2.1 & 6.3 & 15 & -8.2 & 3.2 \\
\hline 1910 & 180 & 211.1 & 209.4 & 172.8 & 773.4 & 12.2 & -2 & 22.5 & 6.5 & 9.1 \\
\hline 1911 & 189.6 & 191.6 & 103.5 & 104.2 & 588.9 & 18.1 & -11 & -39.5 & -35.8 & -17 \\
\hline 1912 & 144.8 & 253.3 & 196.6 & 142.3 & 737.1 & -9.4 & 16.8 & 15.2 & -13.1 & 3.6 \\
\hline 1913 & 133.9 & 228.8 & 98.8 & 130.9 & 592.5 & -15.9 & 5.7 & -42.3 & -20 & -16.6 \\
\hline 1914 & 157.6 & 280.4 & 211.6 & 186.5 & 836.1 & -1.3 & 29.3 & 24.2 & 13.9 & 17.7 \\
\hline
\end{tabular}


International Journal of Innovative Technology and Exploring Engineering (IJITEE) ISSN: 2278-3075, Volume-9 Issue-2S3, December 2019

\begin{tabular}{|c|c|c|c|c|c|c|c|c|c|c|}
\hline 1915 & 193.8 & 214.5 & 149.5 & 198.2 & 755.9 & 21.6 & -1.1 & -12.4 & 21.3 & 6.4 \\
\hline 1916 & 199.6 & 248.9 & 183.7 & 190.3 & 822.6 & 25.4 & 15.1 & 8.1 & 17.1 & 16.2 \\
\hline 1917 & 206.4 & 157.1 & 198.1 & 246.8 & 808.3 & 29.6 & -27.4 & 16.6 & $\begin{array}{l}51.8 \\
\end{array}$ & 14.2 \\
\hline 1918 & 114.4 & 85.5 & 123.7 & 110.4 & 434 & -28.1 & -60.5 & -27.2 & -32.1 & -38.7 \\
\hline 1919 & 176.2 & 196.8 & 127.6 & 191.5 & 692.1 & 10.8 & $\begin{array}{l}-8.9 \\
\end{array}$ & -24.8 & 18.1 & -2.1 \\
\hline 1920 & 169 & 192.5 & 127.5 & 136.3 & 625.3 & 6.2 & -10.9 & -24.9 & -15.9 & -11.6 \\
\hline 1921 & 141.7 & 282.9 & 184.5 & 133.2 & 742.3 & -10.9 & 30.9 & 8.7 & -17.9 & 5 \\
\hline 1922 & 146.8 & 224.8 & 129.8 & 109.9 & 611.3 & -6.5 & 4.2 & -23.5 & -32.3 & -13.3 \\
\hline 1923 & 119.6 & 295.5 & 171.6 & 183.1 & 769.8 & -24.7 & 36.6 & 1.4 & 12.6 & 8.9 \\
\hline 1924 & 161.6 & 313.9 & 176.9 & 212.7 & 865.2 & 1.8 & 45.1 & 4 & 30.5 & 22.2 \\
\hline 1925 & 167.1 & 212.6 & 191 & 100.2 & 670.9 & 5.6 & -1.6 & 11.7 & -38.4 & -5.2 \\
\hline 1926 & 116.3 & 231.3 & 183.5 & 163.2 & 694.3 & -27.1 & 6 & 6.7 & 0.4 & -2.5 \\
\hline 1927 & 180.9 & 238.9 & 140.4 & 178.6 & 738.8 & 13.4 & 9.5 & -18.4 & 10.1 & $\begin{array}{l}3.8 \\
\end{array}$ \\
\hline 1928 & 153.8 & 190.9 & 173.2 & 132.5 & 650.4 & -3.9 & -12.5 & 0.6 & -18.3 & -8.7 \\
\hline 1929 & 207.1 & 161.7 & 119.6 & 179.2 & 667.7 & 29.9 & -25.6 & -30.3 & 10.8 & $\begin{array}{l}-6 \\
\end{array}$ \\
\hline 1930 & 185.8 & 125.1 & 109.8 & 172.1 & 592.8 & 15.3 & -43.8 & -37.3 & 6.7 & -17.7 \\
\hline 1931 & 157.4 & 220.5 & 229.8 & 170.3 & 778 & -2 & 0.1 & 32.5 & 6.1 & 8.8 \\
\hline 1932 & 107.4 & 230.3 & 191.4 & 148.4 & 677.6 & -33.1 & 4.6 & 10.5 & -7.6 & -5.2 \\
\hline 1933 & 209.4 & 234.9 & 211.7 & 198.1 & 854.1 & 24.5 & 2 & 17.6 & 21.6 & 15.2 \\
\hline 1934 & 187 & 208 & 172.9 & 83.5 & 651.3 & 11.3 & $\begin{array}{l}-9.8 \\
\end{array}$ & -4.1 & -48.9 & -12.2 \\
\hline 1935 & 137 & 218.9 & 190.8 & 147.3 & 693.9 & -18.7 & -5.2 & 6 & $\begin{array}{l}-9.7 \\
\end{array}$ & -6.5 \\
\hline 1936 & 199 & 212.5 & 152.2 & 155 & 718.7 & 18.4 & -7.5 & -15.5 & -4.9 & -3 \\
\hline 1937 & 122.7 & 270.1 & 117.6 & 126.9 & 637.2 & -27.1 & 17.5 & -34.6 & -22.2 & -14 \\
\hline 1938 & 200.1 & 215.1 & 215.6 & 208.7 & 839.6 & 19.2 & -6.3 & 20.3 & 28.2 & 13.5 \\
\hline 1939 & 139.3 & 186 & 180.7 & 121.7 & 627.6 & $\begin{array}{l}-17 \\
\end{array}$ & -18.8 & 0.9 & -25.1 & -15 \\
\hline 1940 & 178.2 & 228.7 & 228.1 & 107.1 & 742.1 & 6.4 & -0.2 & 27.4 & -34.1 & 0.5 \\
\hline 1941 & 176.7 & 148.3 & 156.1 & 171.4 & 652.5 & 5.6 & -35.2 & -12.7 & 5.6 & -11.5 \\
\hline 1942 & 192.6 & 234.5 & 190.7 & 101.1 & 718.9 & 18.6 & 3.5 & 9 & -36.3 & -0.5 \\
\hline 1943 & 172 & 223.9 & 104.1 & 187 & 687.1 & $\begin{array}{l}4.8 \\
\end{array}$ & -2 & -41 & 17.8 & -5.6 \\
\hline 1944 & 145 & 234.9 & 119.4 & 149 & 648.3 & -11 & 3.5 & -32 & -5.9 & -10.4 \\
\hline 1945 & 128.3 & 265.3 & 169.8 & 114.3 & 677.7 & -20.5 & 16.9 & -3.6 & -27.5 & -6.2 \\
\hline 1946 & 200.5 & 219 & 220.5 & 144.9 & 784.9 & 19 & -4.3 & 23.2 & -10.6 & 6.3 \\
\hline 1947 & 133.1 & 252.9 & 262.2 & 202.8 & 851 & -20.4 & 11 & 46.3 & 24.8 & 15.5 \\
\hline 1948 & 163.8 & 219.1 & 206.3 & 147.1 & 736.4 & -2.1 & -4.3 & 15 & -9.7 & -0.3 \\
\hline 1949 & 156.7 & 259.2 & 188.8 & 201.8 & 806.6 & $\begin{array}{l}-7.4 \\
\end{array}$ & 13.2 & 5.7 & 24.3 & 9.1 \\
\hline 1950 & 133.7 & 265.6 & 158.9 & 211.2 & 769.5 & -20.2 & 16 & -11.4 & 30 & 4.2 \\
\hline 1951 & 149.4 & 256.1 & 153 & 140.9 & 699.4 & -11.2 & 10.9 & -15.6 & -12.6 & -5.7 \\
\hline 1952 & 131.1 & 170 & 150 & 77.3 & 528.4 & -21.9 & -26.3 & -16.3 & -51.9 & -28.5 \\
\hline 1953 & 170.1 & 311.8 & 183.6 & 164.4 & 829.8 & 2.9 & 35.7 & 3 & 2.7 & 13.1 \\
\hline 1954 & 158.3 & 282.3 & 204.8 & 143.3 & 788.7 & -3.8 & 25.2 & 15.8 & -10.7 & 8.4 \\
\hline 1955 & 184.8 & $\begin{array}{ll}158 \\
\end{array}$ & 208.7 & 204 & 755.5 & 12.6 & -29.8 & 18.1 & 27.2 & 4 \\
\hline 1956 & 210.8 & 277.1 & 158.8 & 164.5 & 811.2 & 30.7 & 23.2 & -9.9 & 3.1 & 12.4 \\
\hline 1957 & 205.8 & 225.5 & 209.1 & 91.6 & 732 & 24.7 & -0.5 & 18.6 & -42.7 & 0.6 \\
\hline 1958 & 151 & 285 & 258.3 & 119.4 & 813.7 & -8.6 & 24.7 & 46.1 & -25 & 11.5 \\
\hline 1959 & 236.7 & 342.2 & 201.3 & 171.5 & 951.7 & 44.3 & 50.9 & 13.9 & 7.5 & 30.9 \\
\hline 1960 & 153.8 & 237.2 & 112.5 & 228.4 & 732 & -7.8 & 4.1 & -36.4 & 42.8 & 0.1 \\
\hline 1961 & 213.2 & 353.5 & 207.5 & 141.5 & 915.7 & 27.1 & 62.5 & 20.2 & -8.4 & 28.5 \\
\hline 1962 & 92.3 & 256.8 & 227.2 & 208.2 & 784.4 & -45.1 & 18 & 32 & 34.4 & 10.1 \\
\hline 1963 & 145.5 & 192.5 & 240.3 & 111.1 & 689.4 & -11 & -15.1 & 36.5 & -30.4 & -5 \\
\hline 1964 & 124.7 & 252.2 & 220.6 & 246.2 & 843.7 & -24.3 & 12.3 & 25.3 & 52.3 & 16.1 \\
\hline 1965 & 131.9 & 228 & 168.7 & 140 & 668.6 & -19.1 & 0.2 & -4.7 & -12 & -8 \\
\hline 1966 & 112.6 & 234.1 & 138.5 & 192 & 677.3 & -31.7 & 5 & -20.6 & 20.8 & -6.1 \\
\hline 1967 & 154.2 & 281.1 & 156 & 115.8 & 707.1 & -6 & 23.4 & -11.2 & -27.3 & -2.7 \\
\hline 1960 & 153.8 & 237.2 & 112.5 & 228.4 & 732 & -7.8 & 4.1 & -36.4 & 42.8 & 0.1 \\
\hline 1961 & 213.2 & 353.5 & 207.5 & 141.5 & 915.7 & 27.1 & 62.5 & 20.2 & -8.4 & 28.5 \\
\hline
\end{tabular}




\begin{tabular}{|c|c|c|c|c|c|c|c|c|c|c|}
\hline 1962 & 92.3 & 256.8 & 227.2 & 208.2 & 784.4 & -45.1 & 18 & 32 & 34.4 & 10.1 \\
\hline 1963 & 145.5 & 192.5 & 240.3 & 111.1 & 689.4 & -11 & -15.1 & 36.5 & -30.4 & -5 \\
\hline 1964 & 124.7 & 252.2 & 220.6 & 246.2 & 843.7 & -24.3 & 12.3 & 25.3 & $\begin{array}{l}52.3 \\
\end{array}$ & 16.1 \\
\hline 1965 & 131.9 & 228 & 168.7 & 140 & 668.6 & -19.1 & 0.2 & -4.7 & -12 & -8 \\
\hline 1966 & 112.6 & 234.1 & 138.5 & 192 & 677.3 & -31.7 & 5 & -20.6 & 20.8 & -6.1 \\
\hline 1967 & 154.2 & 281.1 & 156 & 115.8 & 707.1 & -6 & 23.4 & -11.2 & -27.3 & -2.7 \\
\hline 1968 & 144.1 & 253.2 & 85.4 & 192.2 & 674.9 & -11.6 & 15.4 & -50.5 & 20.7 & -5.5 \\
\hline 1969 & 118.1 & 240.9 & 168.9 & 132.5 & 660.4 & -25.6 & 10.1 & -2.5 & -16.4 & -6.9 \\
\hline 1970 & 164.6 & 193.4 & 266 & 149.5 & 773.6 & 2.7 & -13.7 & 52 & -6 & 7.7 \\
\hline 1971 & 181.5 & 149.8 & 160.7 & 146.9 & 638.8 & 16 & -32.4 & $\begin{array}{l}-4.8 \\
\end{array}$ & \begin{tabular}{|l|}
-5.3 \\
\end{tabular} & -9 \\
\hline 1972 & 147.8 & 181.5 & 92.6 & 151.3 & 573.3 & -6.7 & -19.4 & -46.6 & -2.7 & -19.6 \\
\hline 1973 & 154.5 & 191 & 226.2 & 114.5 & 686.2 & 0.9 & -10.3 & 37.5 & -25.3 & 0.4 \\
\hline 1974 & 105.9 & 227.6 & 166.9 & 221 & 721.4 & -33.2 & 2.4 & -2.5 & 41.7 & 1.9 \\
\hline 1975 & 204.4 & 245.5 & 225.9 & 246.4 & 922.2 & 28.6 & 9.4 & 31 & 59.4 & 29.8 \\
\hline 1976 & $\begin{array}{l}94.9 \\
\end{array}$ & 257.7 & 221.5 & $\begin{array}{l}93.6 \\
\end{array}$ & 667.6 & -41.8 & 12.4 & 24.6 & -40.8 & $\begin{array}{l}-8.3 \\
\end{array}$ \\
\hline 1977 & 156 & 221.5 & 167.7 & 110.6 & 655.7 & -2.5 & -1.6 & -3.9 & -28.9 & -8.3 \\
\hline 1978 & 208.7 & 264.2 & 224.1 & 163.1 & 860 & -12.1 & 17.2 & 29 & 5 & 20.6 \\
\hline 1979 & 148.5 & 157.3 & 122.6 & 208.7 & 637.1 & -2.7 & -21.4 & -24.2 & 34.4 & -4.9 \\
\hline 1980 & 188.5 & 178.1 & 183.4 & 119.7 & 669.7 & 20.7 & & 10 & -22.5 & -1.5 \\
\hline 1981 & 180.9 & 188.3 & 185.3 & 235 & 789.5 & 20.2 & 1.8 & 17.7 & 51.6 & 21.9 \\
\hline 1982 & 146.9 & 174.1 & 150.4 & 124.8 & 596.2 & -2.4 & -9.1 & -5.9 & -18.9 & -9.1 \\
\hline 1983 & 152.5 & 206.7 & 294.6 & 256.6 & 910.4 & 0.6 & 6 & 83.4 & 67.3 & 37.8 \\
\hline 1984 & 127.7 & 221.8 & 76.2 & 136.9 & 562.5 & -15.8 & 13.8 & -52.4 & -10.9 & -14.8 \\
\hline 1985 & 168 & 149.5 & 146.3 & 99 & 562.8 & 10.7 & -23.6 & -8.6 & -35.4 & -14.8 \\
\hline 1986 & 140 & 119.1 & 202.7 & 132.6 & 594.3 & -7.3 & -38.5 & 28.1 & -13.1 & -9.3 \\
\hline 1987 & 131 & 107.3 & 192.8 & 110.7 & 541.9 & -11 & -47.6 & 19.4 & -28.5 & -18.9 \\
\hline 1988 & 127.8 & 283.5 & 241.1 & 249.5 & 901.8 & -13.1 & 39.2 & 48.5 & 60.7 & 35 \\
\hline 1989 & 178.5 & 312 & 158.4 & 183.8 & 832.7 & $\begin{array}{l}17.1 \\
\end{array}$ & 45.7 & -6.3 & 15.2 & $\begin{array}{l}19.8 \\
\end{array}$ \\
\hline 1990 & 176.5 & 167 & 240.5 & 133.4 & 717.4 & 7.1 & -24.3 & 21.2 & -18.3 & -4 \\
\hline 1991 & 278 & 239.3 & 167.2 & 135.5 & 820 & 69.4 & 2.5 & -9.2 & -16.9 & 10.1 \\
\hline 1992 & 190.9 & 328.3 & 207.7 & 136.9 & 863.8 & 15.8 & 39.4 & 11.4 & -14.7 & 15.6 \\
\hline 1993 & 139.1 & 230.8 & 156.4 & 126.2 & 652.4 & -15.5 & -1.8 & -14.9 & -22.5 & -12.5 \\
\hline 1994 & 167.3 & 273.6 & 175.4 & 88.9 & 705.2 & 2.7 & 17.5 & -4.1 & -44.9 & -4.7 \\
\hline 1995 & 137.7 & 243.6 & 203.1 & 137.7 & 722.2 & -15.5 & 4.4 & 10.7 & -14.2 & -2.5 \\
\hline 1996 & 233.8 & $\begin{array}{l}198.8 \\
\end{array}$ & 239 & 203.9 & 875.5 & 44.2 & -15.2 & 29 & 27.4 & 18 \\
\hline 1997 & 138.1 & 229.7 & 181.2 & 179.7 & 728.7 & -15.4 & $\begin{array}{l}-0.8 \\
\end{array}$ & -1.1 & 12.1 & -1.3 \\
\hline 1998 & 173.9 & 230.9 & 219.9 & 238.7 & 863.4 & 7.9 & 0.4 & 28.5 & 49 & 19.5 \\
\hline 1999 & 148.5 & 214.8 & 147.3 & 118.2 & 628.8 & -8.7 & -6.6 & -18.5 & -25.6 & -14.1 \\
\hline 2000 & 202.4 & 169.7 & 295.5 & 133.8 & 801.3 & 24.5 & -26.2 & 63.4 & -15.9 & 9.4 \\
\hline 2001 & 162.2 & 159.8 & 164.8 & 172.6 & 659.4 & -0.2 & -30.6 & -8.9 & 8.7 & -10 \\
\hline 2002 & 151.7 & 95.8 & 182 & 77.2 & 506.7 & -8 & -59 & -5.1 & -51.8 & -32.5 \\
\hline 2003 & 141.9 & 227.3 & 196 & 82.8 & 648 & -11.5 & 0 & 6.2 & -48.2 & -11.5 \\
\hline 2004 & 143 & 177.6 & 134.6 & 162.1 & 617.4 & -9.9 & -21.1 & -26.2 & 1.1 & -15 \\
\hline 2005 & 141.9 & 286 & 155.8 & 223.3 & 807 & -9.9 & 27.9 & -14.3 & 39.4 & 11.6 \\
\hline 2006 & 158.1 & 153.9 & 176.4 & 196.1 & 684.5 & 0.4 & -31.2 & -2.9 & 22.5 & -5.3 \\
\hline 2007 & 242.5 & 205.6 & 223.1 & 231 & 902.1 & 54 & $\begin{array}{l}-8 \\
\end{array}$ & 22.8 & 44.3 & 24.8 \\
\hline 2008 & 135.6 & 170.8 & 238.5 & 147.7 & 692.6 & -13.9 & -23.6 & 31.3 & -7.8 & -4.2 \\
\hline 2009 & 106.5 & 216.1 & 161 & 198.5 & 682.2 & -32.3 & -3.3 & -11.4 & 24 & -5.6 \\
\hline 2010 & 163.5 & 277.9 & 229.7 & 177 & 848.1 & 3.6 & 24 & 26.6 & 11 & 17.3 \\
\hline 2011 & 156.8 & 213.1 & 219 & 124.3 & 713.1 & \begin{tabular}{|l|}
-1.3 \\
\end{tabular} & $\begin{array}{l}-2.9 \\
\end{array}$ & 21.2 & -20.6 & $\begin{array}{l}-0.3 \\
\end{array}$ \\
\hline 2012 & 113 & 178.7 & 207.3 & 145 & 643.9 & -28.8 & -18.6 & 14.7 & -7.5 & -10 \\
\hline 2013 & 206.1 & 274.6 & 164.9 & 180.1 & 825.6 & 29.8 & 25.1 & -8.7 & 14.9 & 15.4 \\
\hline 2014 & 100.1 & 199.4 & 229.1 & 136.7 & 665.3 & -36.9 & -9.1 & 26.8 & 12.8 & -7 \\
\hline
\end{tabular}




\begin{tabular}{|l|l|l|l|l|l|l|l|l|l|l|}
\hline 2015 & 187.6 & 110.2 & 146.2 & 161 & 605.1 & 18.1 & -49.9 & -19.1 & 2.8 & -15.5 \\
\hline 2016 & 199.1 & 193.3 & 108.7 & 159.2 & 660.2 & 25.3 & -12.1 & -39.8 & 1.7 & -7.8 \\
\hline 2017 & 156.4 & 189.4 & 154.8 & 126.4 & 640.5 & 12.5 & 16.8 & 15.2 & 19.7 & 16.7 \\
\hline 2018 & 126.4 & 158.4 & 123.8 & 111.7 & 634.8 & 14.9 & 18.6 & 27.6 & 22.6 & 19.4 \\
\hline
\end{tabular}

\section{IV.METHODOLOGY}

Flowchart of the Proposed System

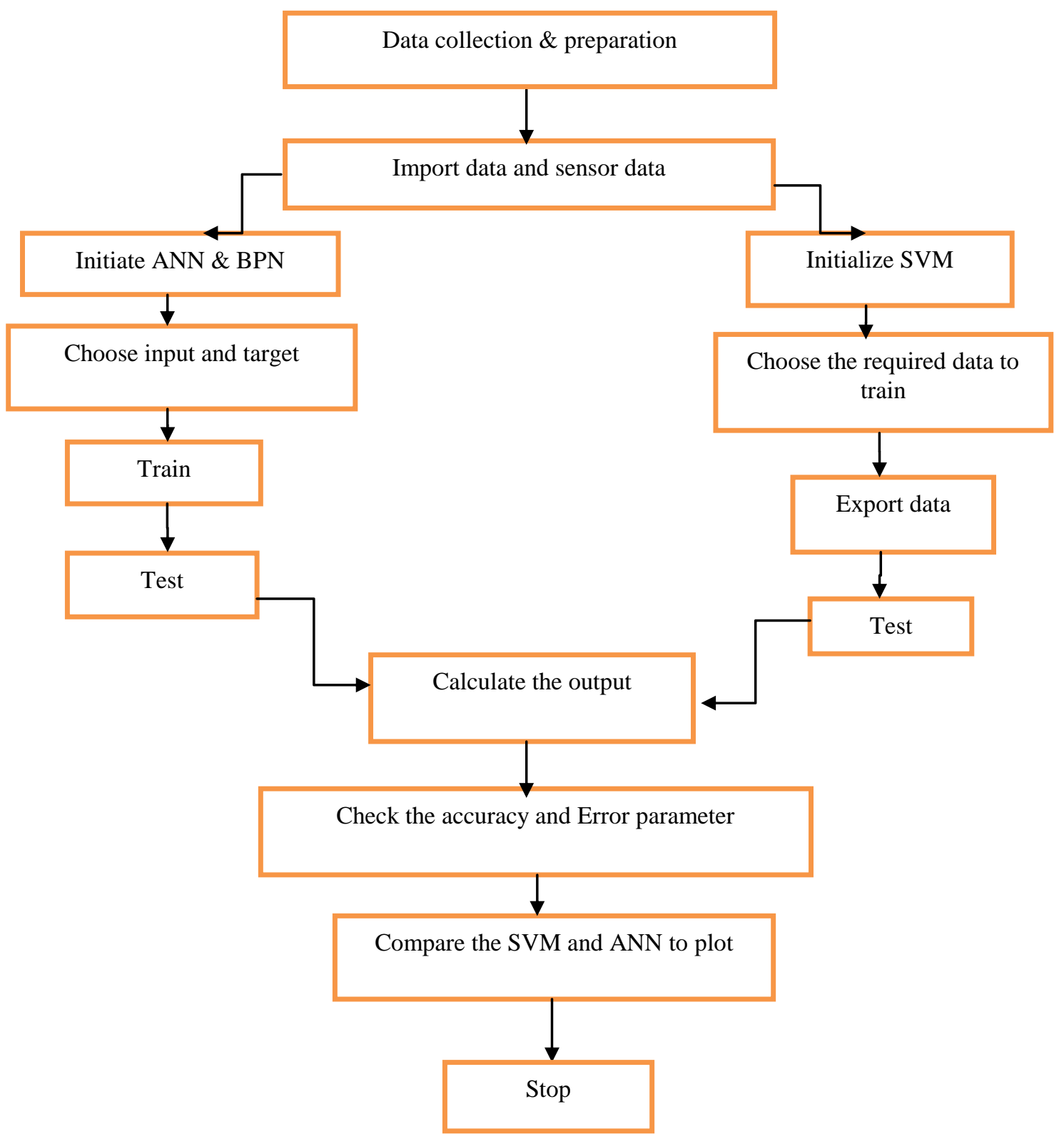

Figure 4.1 Flow diagram of flood prediction using ANN and SVM model

The methods used in this proposed study are Artificial Neural Network (ANN) with Back Propagation Neural Network (BPNN) and Support Vector Machine (SVM). The major dataset are imported to algorithm were trained and tested. The problem defined in flood prediction by calculating rainfall, temperature and water level dataset. In this research, MATLAB tool is used to implement neural network and using classification application for SVM.
The performance evaluation are required to simulate accuracy for flood prediction. The measurement of Root Mean Square Error (RMSE), Mean Absolute Error (MAE) and Coefficient of Determination (R2) is predicted in model. This prediction is compared with ANN and SVM model for better accuracy. 
The neural network and classification application is used to implement flood forecasting. The following implementation work by resulting regression graph in trained, validated and tested values

\section{RAINFALL}

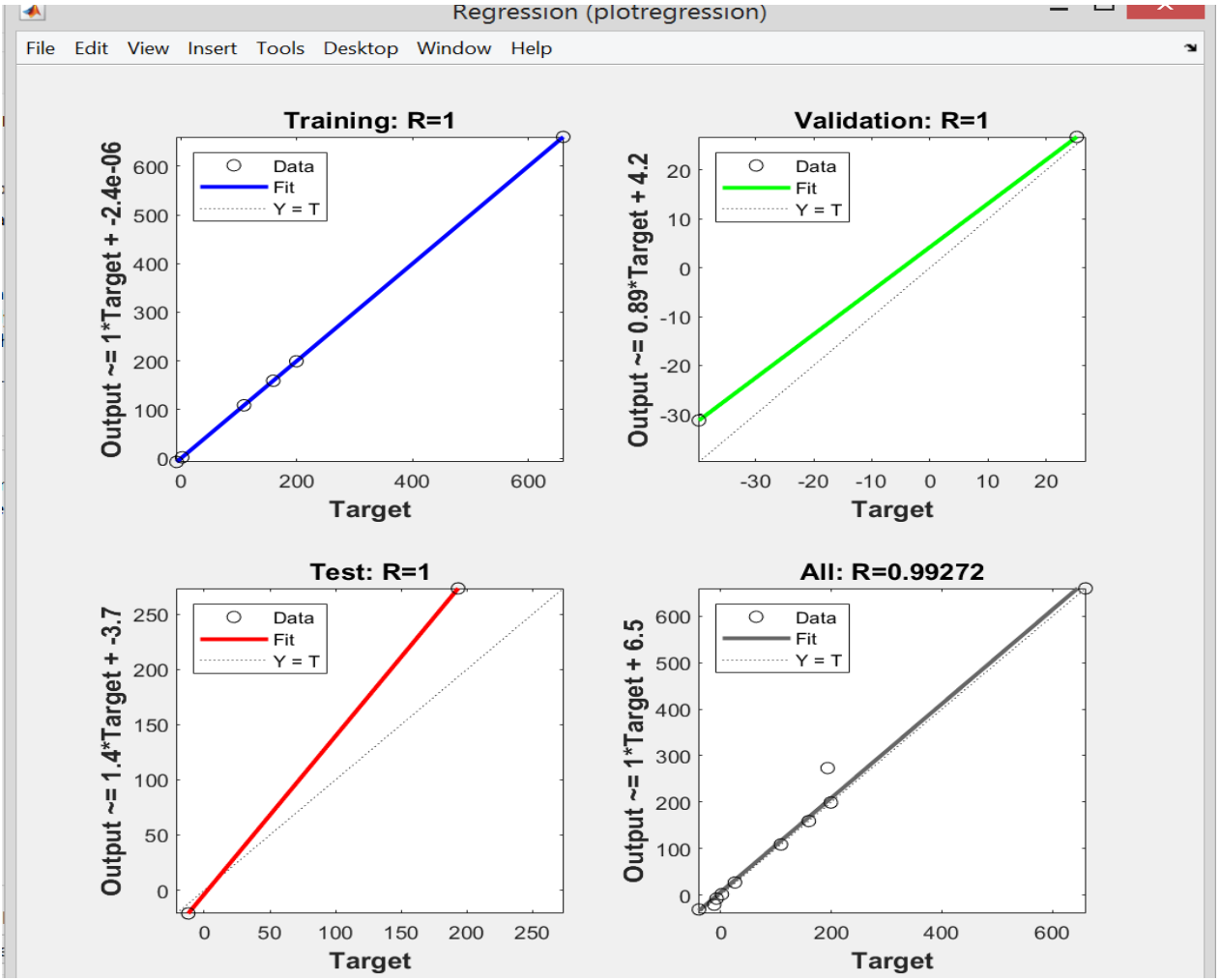

Figure 4.2 Implementing Regression plot in Rainfall data using Neural Network

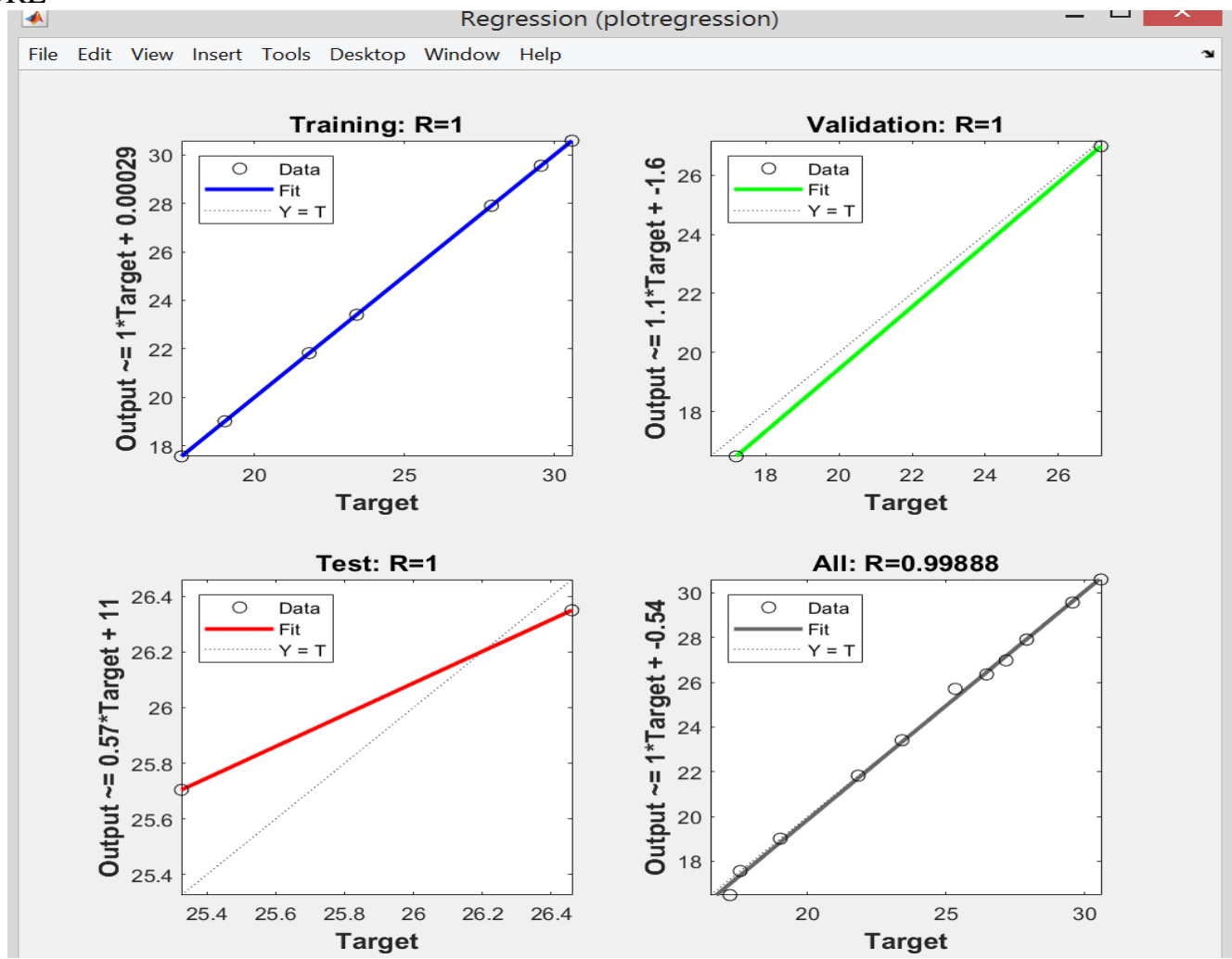

Figure 4.3 Implementing Regression plot in Temperature data using Neural Network 


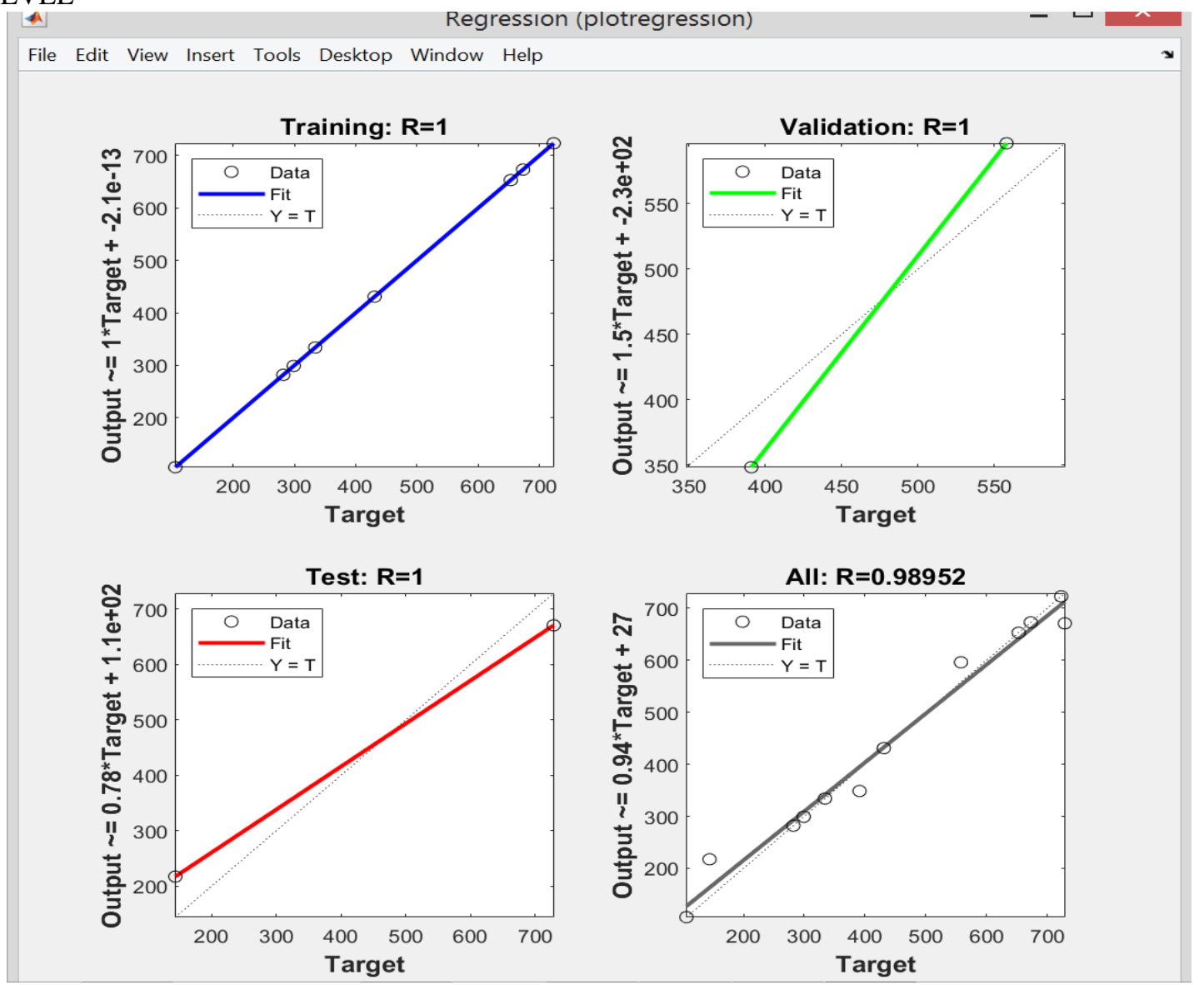

Figure 4.4 Implementing regression plot in trained water level data using Neural Network

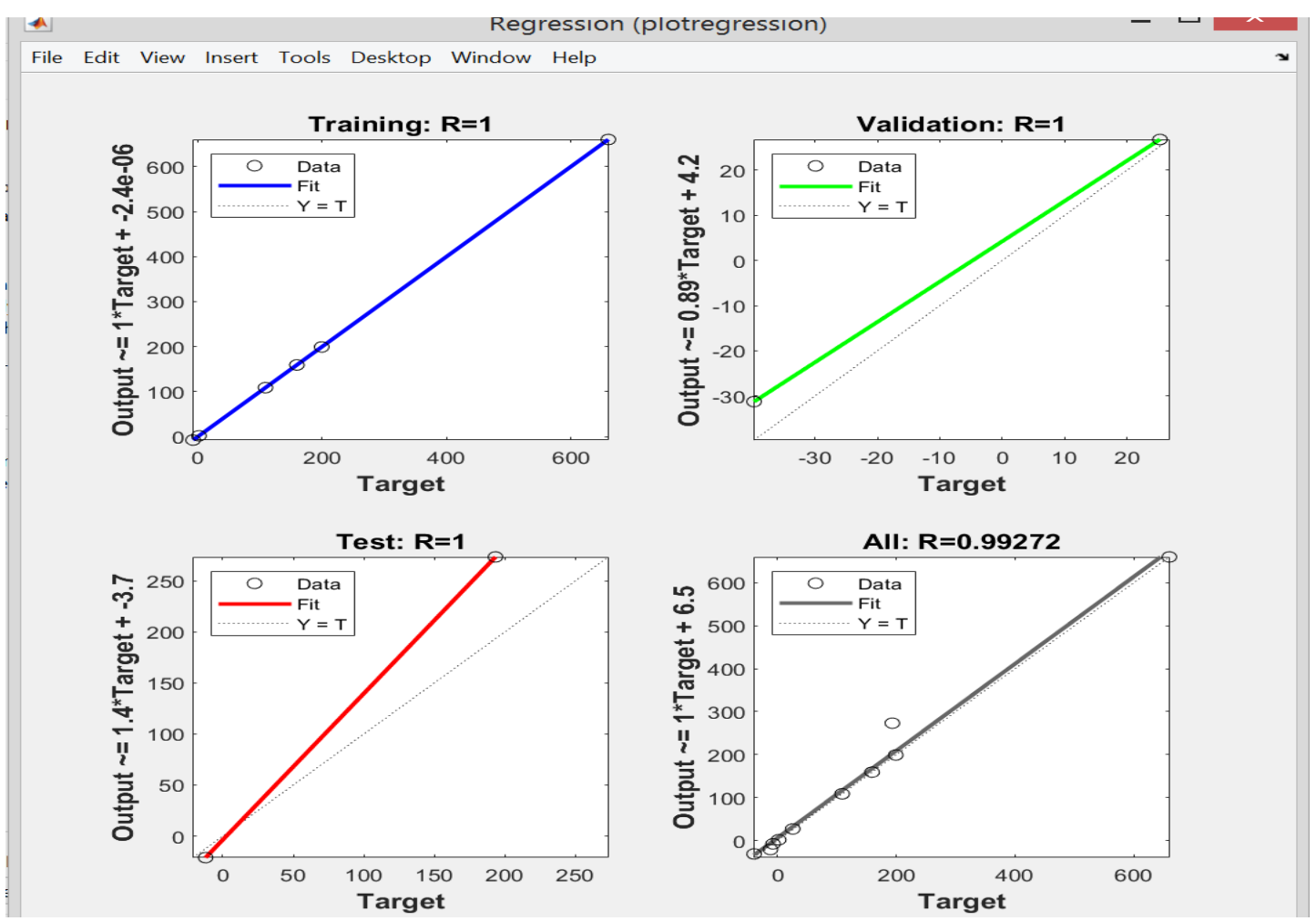

Figure 4.5 Implementing Regression plot in Rainfall data using Neural Network 
The neural network and classification application is used to implement flood forecasting. The following implementation work by resulting regression graph in trained, validated and tested values.

\section{COMPARISON OF ANN AND SVM IN RAINFALL}

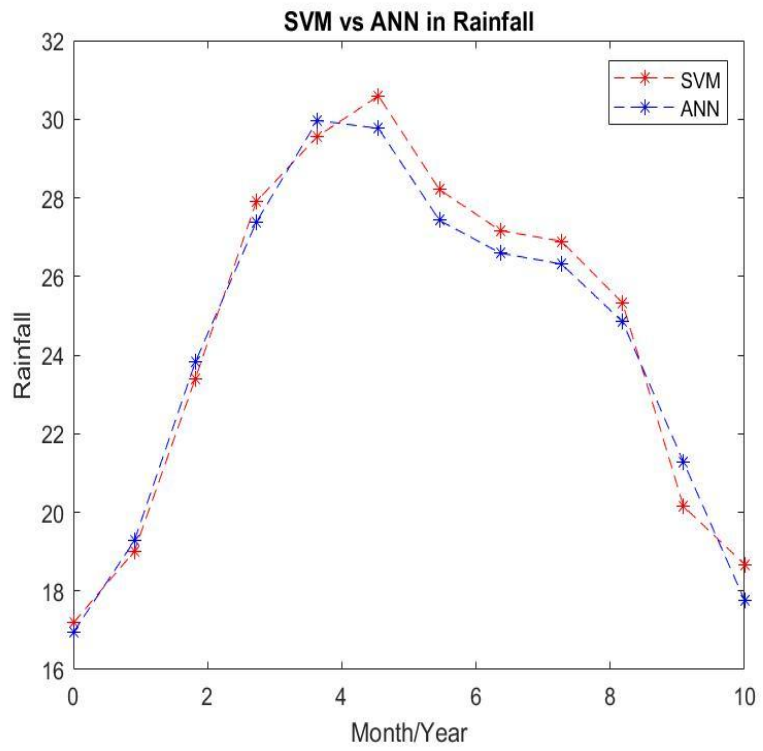

Figure 4.6 Graphical comparison of ANN and SVM model in rainfall data

\section{COMPARISON OF SVM AND ANN TEMPERATURE}

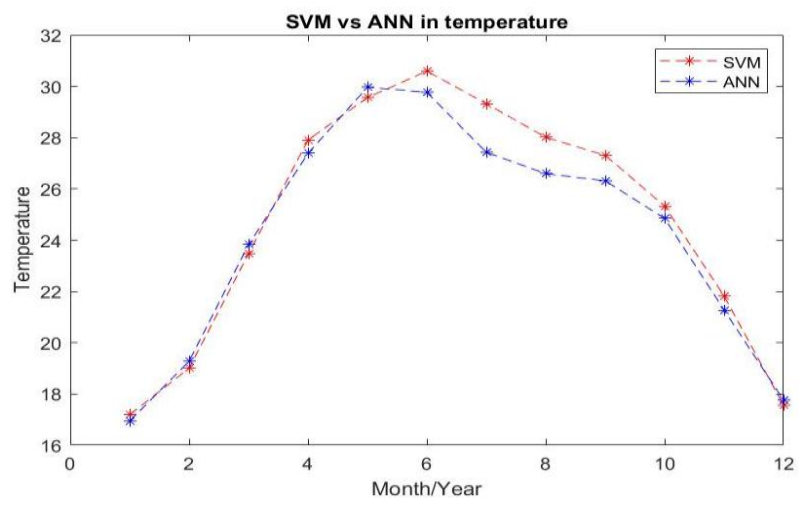

Figure 4.7 Graphical comparison of ANN and SVM model in temperature data

Predicting accuracy of overall dataset between ANN and SVM

\begin{tabular}{|l|l|l|}
\hline ACCURACY & ANN & SVM \\
\hline RAINFALL & 26.1463 & 27.3959 \\
\hline TEMPERATURE & 93.0873 & 95.1226 \\
\hline WATER LEVEL & 88.0225 & 89.5276 \\
\hline
\end{tabular}

\section{CONCLUSION}

An automated flood flow was predicted by proposed system in the region of Korattur lake Chennai, Tamilnadu. The focus of this research is to predict flood with higher accuracy using Artificial Neural Network (ANN) and Support Vector Machine (SVM) algorithm with support of ultrasonic sensor to gather data.Earlier prediction of outflow in water level is done with the help of rainfall, temperature and water level dataset. The predicted dataset is evaluated in terms of Root Mean Square Error (RMSE), Mean Absolute Error
(MAE) and Coefficient of Determination (R2) for the performance in flood forecasting. The comparison of Artificial Neural Network (ANN) and Support Vector Machine (SVM) algorithm results Support Vector Machine (SVM) is more effective compared with Artificial Neural Network (ANN) for flood prediction. This research is an automated software development of proper planning for early evacuation is done by predicting flood.

\section{REFERENCES}

1. H. Shahraiyni, M. Ghafouri, S. Shouraki, B. Saghafian, and M Nasseri, "Comparison between active learning method and support vector machine for runoff modeling," J. Hydrol. Hydromechanics, vol 60 , no. 1, pp. 16-32, 2012.

2. S. H. Elsafi, "Artificial Neural Networks (ANNs) for flood forecasting at Dongola Station in the River Nile, Sudan," Alexandria Eng. J., vol 53, no. 3, pp. 655-662, 2014.

3. J. Noymanee, N. O. Nikitin, and A. V. Kalyuzhnaya, "Urban Pluvial Flood Forecasting using Open Data with Machine Learning Techniques in Pattani Basin," Procedia Comput. Sci., vol. 119, no. 2017, pp. 288-297, 2017.

4. O. I. Abiodun, A. Jantan, A. E. Omolara, K. V. Dada, N. A. E. Mohamed, and H. Arshad, "State-of-the-art in artificial neural network applications: A survey," Heliyon, vol. 4, no. 11, p. e00938, 2018.

5. R. UshaRani, T. K. R. Krishna Rao, and R. Kiran Kumar Reddy, “An Efficient Machine Learning Regression Model for Rainfal Prediction," Int. J. Comput. Appl., vol. 115, no. 23, pp. 24-30, 2015.

6. J. Abbot and J. Marohasy, "Forecasting of Medium-term Rainfall Using Artificial Neural Networks: Case Studies from Eastern Australia," Eng. Math. Top. Rainfall, 2018.

7. F. Liu, F. Xu, and S. Yang, "A Flood Forecasting Model Based on Deep Learning Algorithm via Integrating Stacked Autoencoders with BP Neural Network," Proc. - 2017 IEEE 3rd Int. Conf. Multimed. Big Data, BigMM 2017, pp. 58-61, 2017.

8. M. Hitokoto and M. Sakuraba, "Applicability of the Deep Learning Flood Forecast Model Against the Inexperienced Magnitude of Flood," vol. 3, pp. 901-893, 2018 .

9. D. Hassan, K. Ansari, R. Bano, A. Anwar, N. Shaikh, F. Zaidi, and M. Ali, "Application of Neural Networks for River Flow Forecasting," Int J. Sci. Eng. Res., vol. 8, no. May, pp. 1365-1371, 2017.

10. M. Campolo, A. Soldati, and P. Andreussi, "Artificial neural network approach to flood forecasting in the River Arno," Hydrol. Sci. J., vol 48, no. 3, pp. 381-398, 2003.

11. D. Panagoulia, "Artificial neural networks and high and low flows in various climate regimes," Hydrol. Sci. J., 2006.

12. J. R. Mohanty and M. R. Mohapatra, "Rainfall Prediction Using Support Vector Machine ( SVM )," vol. 20, no. 3, pp. 6-13, 2018.

13. E. Michael and O. Patience, "Flood Prediction In Nigeria Using Artificial Neural Network American Journal of Engineering Research ( AJER )," no. 9, pp. 15-21, 2018.

14. Y. Hasbi, W. Budi, and S. Rukun, "Feed Forward Neural Network Modeling for Rainfall Prediction," E3S Web Conf., vol. 73, p. 05017, 2018.

15. R. Bustami, N. Bessaih, C. Bong, and S. Suhaili, "Artificial Neural Network for Precipitation and Water Level Predictions of Bedup River," Int. J. Comput. Sci., vol. 34, no. 2, pp. 1-9, 2007.

16. Albert Alexander Stonier and Brad Lehman, "An Intelligent Based Fault Tolerant System for Solar Fed Cascaded Multilevel Inverters", IEEE Transactions on Energy Conversion, Vol.33, No.3, pp.1047-1057, September 2018.

\section{AUTHORS PROFILE}

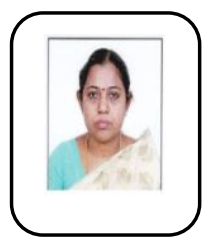

Dr. Savithri.V completed her Under Graduation in Anna Adarsh College, Anna Nagar, Chennai, Master's in Annamalai University, Chidambaram and M.Phil. in Alagappa University, Karaikudi. She acquired Ph.D. Computer Science in 2013 from Mother Teresa Women's University, Kodaikanal. 
She is currently working in the PG Department of Computer Science and Technology, Women's Christian College, Chennai. She has received the National Educational Star Award for excellence in Marvelous Education System and Services to the Nation by GOAL Society, New Delhi and Senior Educator Scientist Award by National Foundation for Entrepreneurship Development, Coimbatore. She has Published 32 International Journals and presented paper in 12 International conferences and one in Book Chapter. She has guided 13 M.Phil. Students. She is a member of various professional bodies like ICTACT, IJCOT, ISJCRESM, etc. Her research area includes Artificial Neural Networks, Medical Imaging,Pattern Recognition and Cloud security.

Ms. Suganya.D pursed Bachelor of Computer Application from University of Madras, Chennai in 2014 and Master of Science from University of Madras in year 2017. She is currently pursuing M.Phil (Computer Science)in Department of Computer Sciences, men's Christian College, University of Madras, Chennai. Her main research work focuses on Machine Learning, Artificial Intelligence. 